Canadian Journal of Physics

Canadian

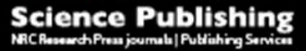

Revue canadienne de physique

\title{
CALCULATION OF THE TILT ANGLE AND SUSCEPTIBILITY AS FUNCTIONS OF TEMPERATURE AND THE ELECTRIC FIELD CLOSE TO THE Sm A - Sm C* TRANSITION IN C7
}

\begin{tabular}{|r|l|}
\hline Journal: & Canadian Journal of Physics \\
\hline Manuscript ID & cjp-2017-0731.R1 \\
\hline Manuscript Type: & Article \\
\hline Date Submitted by the Author: & 11 -Dec-2017 \\
\hline Complete List of Authors: & $\begin{array}{l}\text { Aksoy, S.; Canakkale Onsekiz Mart Universitesi, physics } \\
\text { Kurtseven, H.; Orta Dogu Teknik Universitesi Fen Edebiyat Fakultesi } \\
\text { Kurt, M.; Canakkale Onsekiz Mart Universitesi }\end{array}$ \\
\hline $\begin{array}{r}\text { Is the invited manuscript for } \\
\text { consideration in a Special } \\
\text { Issue? : }\end{array}$ & $\begin{array}{l}\text { Tilt angle, Susceptibility, Smectic A- Smectic C* transition, C7, Landau } \\
\text { model }\end{array}$ \\
\hline \multicolumn{2}{|c|}{} \\
\hline
\end{tabular}


CALCULATION OF THE TILT ANGLE AND SUSCEPTIBILITY AS FUNCTIONS OF TEMPERATURE AND THE ELECTRIC FIELD CLOSE TO THE Sm A - Sm C* TRANSITION IN C7

\author{
Aksoy S. ${ }^{1}$, Yurtseven H. $^{2}$, Kurt M. ${ }^{1}$ \\ ${ }^{1}$ Department of Physics, 18 Mart University, 17100 Çanakkale TURKEY \\ ${ }^{2}$ Department of Physics, Middle East Technical University, 06531 Ankara TURKEY
}

\title{
ABSTRACT
}

Smectic A-Smectic $\mathrm{C}^{*}$ transition in the ferroelectric liquid crystal (C7) is studied as functions of the electric field and the temperature. By expanding the free energy in terms of the tilt angle $\Theta$ (smectic $\mathrm{C}^{*}$ phase) and the polarization $\mathrm{P}$ with a linear coupling between $\Theta$ and $\mathrm{P}(\mathrm{P} \Theta)$ in the mean field theory, the tilt angle is calculated as a function of the electric field $(\Theta$ vs. E) at constant temperatures with respect to the transition temperature for $\mathrm{C} 7 \mathrm{using}$ the observed data from the literature. Also, by extracting the tilt angle from the observed electric displacements at various temperatures (at constant field strength), the inverse tilt angle susceptibility $\left(\chi_{\theta}^{-1}\right)$ derived from the free energy, is fitted to the observed data for the dielectric constant and the fitted parameters are determined. Our calculated $\Theta$ and $\chi_{\theta}$ show that the mean field theory explains adequately the observed behavior of the Smectic A- Smectic $\mathrm{C}^{*}$ transition in the liquid crystals $\mathrm{C} 7$.

Key Words: Tilt angle. Susceptibility. Smectic A- Smectic C* transition. C7.

\section{INTRODUCTION}

Smectic A-ferroelectric-smectic C $\left(\mathrm{C}^{*}\right)$ transition has been studied extensively using various experimental techniques and some theoretical models. Under the electric field applied, in particular, the $\mathrm{Sm} \mathrm{A-Sm} \mathrm{C}\left(\mathrm{C}^{*}\right)$ transition of a first order has also been studied previously as reported in the literature [111]. Recently, ferroelectricity in liquid crystals with the experimental studies has been reviewed [12] and more recent studies in liquid crystal research in general have been given [13] in the literature.

Smectic liquid crystal phases exhibit in the presence of chiral molecules a coupling between the electric polarization $(\mathrm{P})$ and the orientation of the director tilted by an angle $\Theta$ with respect to the normal vector of the smectic layer planes [4]. If the constituent molecules are optically active the Sm $\mathrm{C}^{*}$ phase will be observed instead of the Sm C phase [14]. As a consequence of the molecular chirality the director builds up a helical superstructure by processing around the layer normal with the helical pitch in the Sm $\mathrm{C}^{*}$ phase [3]. The smectic $\mathrm{C}^{*}$ phase is similar to the smectic A phase, the molecules are also packed in layers, but the director is tilted with respect to the layer normal and rotates around the direction perpendicular to the smectic layer with the director tilt angle $\Theta$ as an order parameter [15] as stated above.

$\mathrm{AC}\left(\right.$ or $\mathrm{AC}^{*}$ ) transitions are usually second order but some observations on ferroelectric compounds with high spontaneous polarizations (A7, C7 etc.) have demonstrated the possibility of existence of first order $\mathrm{AC}^{*}$ transitions [16, 17], as also pointed out previously [18]. It has been demonstrated experimentally in the case of A7 that the smaller temperature range of its Sm A phase leads to a weakly first order $\mathrm{AC}^{*}$ transition [19].

In order to describe the nature of the Sm A- Sm $\mathrm{C}^{*}$ transition (first order or second order) in liquid crystals, mean field models have been employed with bilinear PӨ coupling [20,21] and with the biquadratic $\mathrm{P}^{2} \Theta^{2}$ coupling [22-24]. We have also used Landau mean field models in our earlier studies [6$11,24]$.

In this study, we calculate the tilt angle $(\Theta)$ and the tilt susceptibility $\left(\chi_{\theta}\right)$ as functions of temperature and the electric field for the Sm A-Sm $\mathrm{C}^{*}$ transition in $\mathrm{C} 7$. For this calculation, we use the Landau phenomenological model by expanding the free energy in terms of the order parameters (tilt angle $\Theta$ and the spontaneous polarization $\mathrm{P}$ ) with the bilinear coupling ( $\mathrm{PO}$ coupling) in the presence of an applied electric field (E). The observed data [3] are used for the Sm A-Sm C ${ }^{*}$ transition in C7 as a ferroelectric liquid crystal and a first order transition is considered up to $\approx 50 \mathrm{kV} / \mathrm{cm}$ applied electric field $\left(T_{C} \approx 55.8{ }^{\circ} \mathrm{C}\right)$ for this compound. Above this electric field, a second order transition occurs in $\mathrm{C} 7$ on the basis of the experimental measurements [3].

Below, in section 2 we give an outline of the Landau mean field theory. We give our calculations and results in section 3. Sections 4 and 5 give our discussion and conclusions, respectively. 


\section{THEORY}

In the Landau phenomenological model, the free energy density can be written in terms of the order parameters $\theta$ (tilt angle) and $\mathrm{P}$ (polarization) with a bilinear coupling $P \theta$ between $\theta$ and $\mathrm{P}$ to describe the $\mathrm{Sm} \mathrm{A}$ - ferroelectric-Sm C transition ( $\mathrm{Sm} \mathrm{C}^{*}$ ) in liquid crystals in the presence of the electric field $\mathrm{E}$ as also given previously [3],

$$
g=g_{0}+\frac{1}{2} a\left(T-T_{0}\right) \theta^{2}+\frac{1}{4} b \theta^{4}+\frac{1}{6} c \theta^{6}+\frac{1}{2 \chi_{0} \varepsilon_{0}} P^{2}-C P \theta-E P
$$

In Eq. (1), $T_{0}$ denotes the high - temperature limit when coupling constant $C=0$ and $E=0, \varepsilon_{0}$ is the electric permittivity, $\chi_{0}$ is the susceptibility at a constant tilt angle $\left(\theta=\theta_{0}\right)$ and the constants $a>0$, $c>0$ and $b<0$ for the first order and $b>0$ for the second order transitions.

By minimizing the free energy with respect to the polarization $(d g / d P=0)$, polarization can be related to the tilt angle as

$$
P=(C \theta+E) \chi_{0} \varepsilon_{0}
$$

Also, minimizing the free energy with respect to the tilt angle $(d g / d \theta=0)$ and substituting the Eq.(2) into the minimized energy with the definition of $\alpha=a\left(T-T_{0}\right)$ gives

$$
\left(\alpha-C^{2} \chi_{0} \varepsilon_{0}\right) \theta+b \theta^{3}-c \theta^{5}-C E \chi_{0} \varepsilon_{0}=0
$$

The tilt angle $\theta$ can be solved from Eq. (3). The solution of Eq. (3) can be simplified in the absence of the coupling constant $(\mathrm{C}=0)$. By defining $\theta^{2}=x$, the solutions then become

$$
x_{1,2}=-\frac{b}{2 c} \pm \frac{b}{2 c} \sqrt{1-\frac{4 \alpha c}{b^{2}}}
$$

In the presence of the coupling constant, Eq. (1) can be modified through Eq. (2) which gives

where

$$
g=g_{0}+\frac{1}{2} a^{*} \theta^{2}+\frac{1}{4} b \theta^{4}+\frac{1}{6} c \theta^{6}
$$

$$
a^{*}=\alpha+2 C^{2}\left(\chi_{0} \varepsilon_{0}-1\right)
$$

As the transition occurs from Sm $\mathrm{C}^{*}$ to Sm A with increasing temperature, at $T_{c}$ when $\theta=0$ in the zero field $(E=0)$, solution of Eq. (5) can be obtained in the same form as Eq. (4) with the replacement of $\alpha$ by $a^{*}$. This gives rise to the critical temperature $T_{c}$ as

$$
T_{c}=T_{0}+\frac{3 b^{2}}{16 a c}-\frac{C^{2}}{a}\left(2 \chi_{0} \varepsilon_{0}-1\right)
$$

In the absence of the coupling constant $(\mathrm{C}=0)$, the temperature difference $T_{c}-T_{0}$ for a first order $\mathrm{Sm} \mathrm{A}-$

$\mathrm{Sm} \mathrm{C}$ * transition is reduced to $\frac{3 b^{2}}{16 a c}$, which is obtained at zero electric field within the framework of the Landau phenomenological model.

The electric field $\mathrm{E}$ can be obtained as a function of temperature and tilt angle $\theta$ by minimizing the free energy density $(d g / d \theta=0)$ from Eq. (1), which can be written as

$$
E=\frac{1}{C \chi_{0} \varepsilon_{0}}\left\{\left[a\left(T-T_{0}\right)-C^{2} \chi_{0} \varepsilon_{0}\right] \theta+b \theta^{3}+c \theta^{5}\right\}
$$

for the Sm A - ferroelectric-Sm C transition [3]. It has been indicated that the $\theta-E$ curves are in S-like shapes for a discontinuous (first order) transition $(b<0)$, whereas this transition becomes second order (continuous) at $T=T_{c}(\mathrm{~b}>0)[3]$.

The electric displacement $\mathrm{D}$ can be calculated from the tilt angle $\theta$ according to [3]

$$
D=P+\varepsilon_{0} E
$$

with Eq.(2) or from the values of the electric field displacement, the tilt-angle values can be calculated as 


$$
\theta=\frac{1}{\chi_{0} \varepsilon_{0} C}\left[D-\varepsilon_{0} E\left(1+\chi_{0}\right)\right]
$$

Thus, by knowing the temperature dependence of the displacement $\mathrm{D}$, the tilt angle $\theta$ can be calculated as a function of temperature at constant electric fields for the smectic $\mathrm{A}-$ smectic $\mathrm{C}^{*}$ transition in liquid crystals.

Finally, the temperature dependence of the inverse susceptibility for the order parameter (tilt angle $\theta$ ) can be calculated as a function of temperature at a constant electric field $\mathrm{E}$ according to the $\chi_{\theta}^{-1}=\partial E / \partial \theta$ which gives from Eq. (8)

$$
\chi_{\theta}^{-1}=\frac{1}{C \chi_{0} \varepsilon_{0}}\left\{\left[a\left(T-T_{0}\right)-C^{2} \chi_{0} \varepsilon_{0}\right]+3 b \theta^{2}+5 c \theta^{4}\right\}
$$

by means of Eq. (10) using the temperature dependence of the displacement D.

\section{CALCULATIONS AND RESULTS}

The tilt angle $(\theta)$ was analyzed at various filed strengths according to Eq. (8) as analyzed using the experimental data for $\mathrm{C} 7$ [3]. In our analysis, we fitted Eq. (8) to the experimental data for each curve $(a \rightarrow g)$ as shown in Fig.1 with the fitted parameters (Table 1), differently from the previous analysis where the same parameters were used for all the curves [3]. By fitting Eq. (10) to the experimental data for the electric displacement (D) versus the field strength (E) [3] and using the values of the tilt angle ( $\theta$ ), the field strength dependence of the tilt angle was recalculated for C7. Fig.2 gives electric displacement (D) as a function of the field strength (E) using the experimental data [3] according to Eq. (10). With our calculated values of $\mathrm{D}$, we also give the experimental data [3] in this figure. Our calculated $\Theta$ (tilt angle) values are given in Fig. 3 as a function of $\mathrm{E}$ (field strength). For this calculation of D (Fig. 2) and $\Theta$ (Fig. 3 ), a constant $\mathrm{C}$ value of $8.58 \times 10^{7} \mathrm{JC}^{-1} \mathrm{~m}^{-1} \mathrm{rad}^{-1}$ was used for all the curves $(\mathrm{a} \rightarrow \mathrm{e})$.

In order to calculate the temperature dependence of the order parameter susceptibility $\left(\chi_{\theta}\right)$ or the static dielectric constant $(\epsilon)$ at constant field strength $(E)$ for $C 7$, we used the experimental data for the electric displacement (D) versus temperature at constant values of $E=10,30$ and $50 \mathrm{kV} / \mathrm{cm} \mathrm{[3].} \mathrm{We}$ assume the temperature dependence of the electric displacement (D) above and below $\mathrm{T}_{\mathrm{C}}$ at constant field strengths in the quadratic form as

$$
D(T)=a^{\prime}+b^{\prime} T+c^{\prime} T^{2}
$$

with the constants of $a^{\prime}, b^{\prime}$ and $c^{\prime}$, which were determined (Table 2) using the experimental data for D versus $\mathrm{T}$ for $\mathrm{C} 7$ in the vicinity of $\mathrm{T}_{\mathrm{C}}[3]$. The temperature dependence of the tilt angle $\Theta$ was then calculated for constant field strengths (E) studied according to Eq. (10) using the experimental D versus T data for $\mathrm{C} 7$ [3]. By using our calculated $\Theta$ values in the expression for the inverse susceptibility $\left(\chi_{\theta}^{-1}\right)$ in Eq. (11) which was derived from Eq.(8), Eq.(11) was then fitted to the experimental data for the static dielectric constant $\epsilon[3]$ using the definition

$$
\chi_{\theta}^{-1}=1 /(\in-1)
$$

Fig. 4 gives the electric displacement (D) as a function of temperature at constant field strengths $(E=10$, 30 and $50 \mathrm{kV} / \mathrm{cm}$ ) from our analysis (Eq. 12) for C7. We plot in Fig. 5 the static dielectric constant $(\epsilon)$ at various temperatures for constant field strengths according to Eq.(11) through Eq.(13) for C7.

\section{DISCUSSION}

Landau phenomenological model was used in the presence of the electric field (Eq.1) to calculate the tilt angle (order parameter) as functions of temperature and the field strength, and also susceptibility (dielectric constant) at various temperatures (at constant E) for the first order Smectic A - Smectic C* transition in C7. As the field strength increases discontinuity (first order) in the tilt angle $\theta$ (Figs.1 and 3) and in the displacement D (Fig.2) decreases, towards a more continuous (second order) transition from the $\mathrm{Sm} \mathrm{A}$ to Sm C* phase in C7. This occurs at nearly $50 \mathrm{kV} / \mathrm{cm}$ curve e in Fig. 1 and curve $\mathrm{d}$ in Figs. 2 and 3. Below this field strength discontinuity (curves $f$ and $g$ in Fig.1; curves a, b and c in Figs.2 and 3) in the tilt angle and in the displacement is more pronounced and it shifts to lower field strength as also pointed out previously [3]. Above this critical field strength $(\sim 50 \mathrm{kV} / \mathrm{cm})$, continuity (curves $\mathrm{a} \rightarrow \mathrm{d}$ in Fig.1, curve e in Figs.2 and 3) in the tilt angle and in the displacement, occurs for the Sm A - Sm C* transition in $\mathrm{C} 7$, as also observed experimentally [3]. Note that there is a deviation from the linear 
variation of the tilt angle $\theta$ with the field strength $\mathrm{E}$ (curves a and $\mathrm{b}$ in Fig.1) to the nonlinear behavior (curves $\mathrm{c} \rightarrow \mathrm{g}$ in Fig.1) as also shown previously [3]. The critical temperature between discontinuity and continuity was also estimated as $T_{c} \approx 55.8^{\circ} \mathrm{C}$ for $\mathrm{C} 7$ [3]. As an electric-field induced transition, this is most likely weakly first order or nearly second order transition between the smectic phases of $\mathrm{A}$ and $\mathrm{C}^{*}$ in C7. In Fig. 1 for curves (a) and (b), the Sm A-Sm C* transition essentially does not occur. The actual Sm A-Sm C ${ }^{*}$ transition starts with the differences to $\mathrm{T}_{0}$ from curves $\mathrm{c}\left(\mathrm{T}_{0}+1.1{ }^{0} \mathrm{C}\right)$ up to curve $\mathrm{g}\left(\mathrm{T}_{0}+0.3\right.$ ${ }^{0} \mathrm{C}$ ) as the transition temperature $\mathrm{T}_{0}$ is approached. So, linear (curve a) and almost linear nearly quadratic (curve $b$ ) variation of the tilt angle $\Theta$ with the field strength $E$ give rise to considerably different values for the fitted parameters of $b=382 \mathrm{Jm}^{-3} \mathrm{rad}^{-4}$ (very large) and $\mathrm{c}=-5.4 \times 10^{4} \mathrm{Jm}^{-3} \mathrm{rad}^{-6}$ (very small) for curve a, and $b=-8.88 \mathrm{Jm}^{-3} \mathrm{rad}^{-6}$ (small) for curve $\mathrm{b}$, as compared with those values of the fitted parameters of $\mathrm{a}, \mathrm{b}$ and $\mathrm{c}$ for the curves of $\mathrm{c}, \mathrm{d}$, e, f and $\mathrm{g}$ (Table 1), which represent the Sm A-Sm C $\mathrm{S}^{*}$ transition in C7. On the other hand, the value of $b=-23.24 \mathrm{Jm}^{-3} \mathrm{rad}^{-4}$ for curve $b$ (Table 1) indicates that the Sm A-Sm $\mathrm{C}^{*}$ transition may just start from curve $\mathrm{b}$ with the temperature differences of $\mathrm{T}_{0}+1.3{ }^{0} \mathrm{C}$. When we fitted Eq.(8) to the experimental data [3], we assume that the Sm A-Sm C $\mathrm{C}^{*}$ transition occurs for all the curves $(\mathrm{a} \rightarrow \mathrm{g})$ with the transition temperature $\mathrm{T}_{0}$ which increases systematically from $51.66{ }^{\circ} \mathrm{C}$ (curve g) to $54.46{ }^{\circ} \mathrm{C}$ (curve a) as given in Table 1. We determined the fitting parameters $\mathrm{a}, \mathrm{b}, \mathrm{c}$ and the coupling constant $\mathrm{C}$ for all the curves $(\mathrm{a} \rightarrow \mathrm{g})$ where Eq.(8) was fitted as Bahr and Heppke performed to the experimental data [3] for the Sm A-Sm C $\mathrm{C}^{*}$ transition in C7 (Table 1) as stated above. In the present study, each curve was fitted with its own parameters whereas Bahr and Heppke [3] determined the fitting parameters which were used for all the curves $(\mathrm{a} \rightarrow \mathrm{g})$ as given in Table 1 . However, the same fitting parameters for all the curves $(\mathrm{a} \rightarrow \mathrm{g})$ would result in some discrepancies in comparison with the observed data [3]. This was the reason why we fitted Eq.(8) to the experimental data [3] for each curve separately and then we determined the fitting parameters with this fitting procedure, we were able to analyze the experimental data in order to describe the $\mathrm{Sm} \mathrm{A}-\mathrm{Sm} \mathrm{C} \mathrm{C}^{*}$ transition in $\mathrm{C} 7$ adequately. In our fitting procedure for the $\Theta$ versus $\mathrm{E}$ plots, the fitting parameters were determined according to $a>0, b<0$ and $c>0$ for the first order Sm A-Sm C ${ }^{*}$ transition in $\mathrm{C} 7$, as also obtained by Bahr and Heppke [3] with the parameters as given in Table 1. Our values of a and the coupling constant $\mathrm{C}$ can be compared with those of Bahr and Heppke (Table 1). Fitting all the curves $(\mathrm{a} \rightarrow \mathrm{g})$ separately with the close values of the parameters as we determined (Table 1) here, gave us different values compared with those obtained by Bahr and Heppke [3] who used one set of parameters for all the curves. Additionally, we considered the transition temperature $\mathrm{T}_{0}$ describing from the curve a to g systematically for the Sm A-Sm C $\mathrm{C}^{*}$ transition in C7, whereas Bahr and Heppke [3] took only one transition temperature of $\mathrm{T}_{0}=51.36{ }^{\circ} \mathrm{C}$ for this transition. Consequently, different values of the fitting parameters are expected for the two different fitting procedures although the same experimental data [3] were used to analyze the Sm A-Sm C ${ }^{*}$ transition in C7. Bahr and Heppke [3] fitted Eq.(8) to their experimental data for the tilt angle $\Theta$ versus field strength $\mathrm{E}$ with the same fit parameters $(\mathrm{a}, \mathrm{b}, \mathrm{c}$ and $\mathrm{C})$ for all the curves $(\mathrm{a} \rightarrow \mathrm{g})$ as stated above and their plots were good except the curves of $\mathrm{f}$ and $\mathrm{g}$, in particular, which were not in agreement with the observed data. By fitting Eq.(8) to each curve $(\mathrm{a} \rightarrow \mathrm{g})$ with its own fitting parameters (Table 1), we were able to get all the plots which describe the Sm A-Sm $\mathrm{C}^{*}$ transition in $\mathrm{C}$, as given in Fig.1. This is also clearly seen for the curves of a $\rightarrow$ e in Fig.3 which show the $\mathrm{Sm} \mathrm{A-Sm} \mathrm{C}^{*}$ transition in C7. Calculated values of the tilt angle $\Theta$ as a function of the field strength $\mathrm{E}$ according to Eq.(8) (Fig.3) were based on the experimental plot of electric displacement D versus E [3] through Eq.(10) as given in Fig.2. Our calculated plots (D vs. E) are in good agreement with the observed data [3] as shown in Fig.2. However, using the same set of parameters (Table 1) for all the curves $(a \rightarrow e)$ as Bahr and Heppke [3] fitted their experimental data for D versus E [3], was not satisfactory to describe the Sm A-Sm C ${ }^{*}$ transition in C7. Additionally, the temperature dependence of the electric displacement D (Fig.4) and the dielectric constant $\epsilon$ (Fig. 5) was calculated through Eqs. (12) and (11), respectively for constant electric field of 10,30 and $50 \mathrm{kV} / \mathrm{cm}$ by using the experimental data [3] close to the Sm A-Sm $\mathrm{C}^{*}$ transition in $\mathrm{C} 7$. This calculation of $\mathrm{D}$ and $\epsilon$ was based on the experimental plots of $\mathrm{D}$ versus $\mathrm{T}$ and $\epsilon$ versus T, which were not fitted by Bahr and Heppke [3]. As in our plots of $\Theta$-E (Figs. 1 and 3) and D-E (Fig. 2), plots of D-T (Fig. 4) and $\epsilon-\mathrm{T}$ (Fig. 5) were obtained respectively by fitting Eqs. (12) and (13) to the experimental data [3] above and below $\mathrm{T}_{\mathrm{C}}$ for each curve with its fitting parameters (Table 2) for the $\mathrm{Sm} \mathrm{A-Sm} \mathrm{C} \mathrm{C}^{*}$ transition in C7. Also electric displacement $\mathrm{D}$ or the tilt angle $\theta$ decreases with the increasing temperature from the $\mathrm{Sm} \mathrm{A}$ to $\mathrm{Sm} \mathrm{C}^{*}$ phase as the field strength increases from 10 to 50 $\mathrm{kV} / \mathrm{cm}$ for the electric field induced $\mathrm{Sm} \mathrm{A}-\mathrm{Sm} \mathrm{C}$ * transition in C7 (Fig.4).

Discontinuity in the displacement $\mathrm{D}$ (or in the tilt angle $\theta$ ) decreases below the critical field strength $(\sim 50 \mathrm{kV} / \mathrm{cm})$ in the $\mathrm{Sm}$ A phase with increasing temperature toward the critical temperature ( 
$T_{c} \approx 55.8^{\circ} \mathrm{C}$ ) as the field strength increases to $50 \mathrm{kV} / \mathrm{cm}$ as shown in Fig.4. This also indicates that the first order (discontinuous) Sm A - Sm C* transition tends to become a second order (continuous) for C7. The first order nature of the Sm A - Sm C* transition in C7 is more apparent below $50 \mathrm{kV} / \mathrm{cm}$ due to the fact that hysteresis occurs between runs with increasing and decreasing field or temperature within the two-phase region, as observed experimentally [3]. Also, it has been pointed out [3] that because of experimental imperfections (sample impurities and gradients in field strength) instead of a sharp field and temperature value, there occurs a finite field and temperature interval $(\sim 5 \mathrm{kV} / \mathrm{cm}$ and $\sim 0,1 \mathrm{~K}$, respectively) during the crossover from the first order to the second order transition in $\mathrm{C} 7$. On the other hand, the tilt susceptibility $\left(\chi_{\theta}\right)$ or the dielectric constant $(\varepsilon)$ increases with increasing temperature toward the transition temperature from the $\mathrm{Sm} \mathrm{A}$ to the $\mathrm{Sm} \mathrm{C} \mathrm{C}^{*}$ phase and it also increases as the field strength increases from 10 to $50 \mathrm{kV} / \mathrm{cm}$ in C7 (Fig.5). It has been observed that the dielectric constant decreases and broadens when the bias field is increased further up to the $70 \mathrm{kV} / \mathrm{cm}$ [3]. A sharp increase in the dielectric constant (tilt susceptibility) at $50 \mathrm{kV} / \mathrm{cm}$ also indicates crossover from the first order to a second order (divergence like behavior) $\mathrm{Sm} \mathrm{A}-\mathrm{Sm} \mathrm{C*}$ transition in $\mathrm{C} 7$.

\section{CONCLUSIONS}

Temperature and the field strength (E) dependences of the tilt angle $\theta$ (order parameter) and dielectric displacement $\mathrm{D}$ were studied for $\mathrm{Sm} \mathrm{A}-\mathrm{Sm} \mathrm{C}^{*}$ transition in the liquid crystal $\mathrm{C} 7$. The temperature dependence of the tilt susceptibility (dielectric constant) at constant field strengths was also studied for this transition in C7. The Landau phenomenological model with the bilinear coupling between the tilt angle and the polarization was used to describe the first order $\mathrm{Sm} \mathrm{A}-\mathrm{Sm} \mathrm{C}^{*}$ transition in this liquid crystal. By fitting $\theta$ versus $\mathrm{E}$ expression derived from the free energy to the observed data from the literature, temperature and the field strength dependences of the tilt angle, electric displacement and the tilt susceptibility (dielectric constant) were obtained for the $\mathrm{Sm} \mathrm{A}-\mathrm{Sm} \mathrm{C} \mathrm{C}^{*}$ transition in $\mathrm{C}$. Our results show that there is a crossover from the first order to a second order transition with increasing electric field as the discontinuities in the tilt angle and in the electric displacement appearing vanish and in the tilt susceptibility seems to diverge at about $50 \mathrm{kV} / \mathrm{cm}\left(T_{c} \approx 55.8^{\circ} \mathrm{C}\right)$, as observed experimentally. This indicates weakly first order or nearly second order $\mathrm{Sm} \mathrm{A}-\mathrm{Sm} \mathrm{C}$ transition in $\mathrm{C} 7$, which can be described adequately by the Landau phenomenological model as studied here.

\section{REFERENCES}

1. Ch. Bahr, G. Heppke and N.K. Sharma, Ferroelectrics, 76(1987)151.

2. A. Ezcurra, M.A. P. Jubindo, M.R. de La Fuente, J. Etxebarria, A. Remon and M.J. Tello, Liquid Crystals, 4(1989) 125.

3. Ch. Bahr and G. Heppke, Phys. Rev., 41(1990)4335.

4. Ch. Bahr and G. Heppke, Phys. Rev. A, 44(1991)3669.

5. Ch. Bahr, G. Heppke and B. Sabaschus, Liquid Cryst., 11(1992)41.

6. H. Yurtseven, S. Bellici and E. Kilit, Ferroelectrics, 344(2006)83.

7. H. Yurtseven and E. Kilit, Ferroelectrics, 365(2008)122.

8. H. Yurtseven and E. Kilit, Phys. Chem. Liq., 50(2012)367.

9. H.Yurtseven, D. Eren and A. Abdelal, Ferroelectrics, 467(2014)126.

10. H. Yurtseven and T.Mercan, Ferroelectrics, 481(2015)10.

11. M. Kurt and H. Yurtseven, Adv. Condens. Matter Phys., Vol.2012, Article ID 201972, 5 pages.

12. M. Hird, Liq. Cryst., 38(2011)1467.

13. D.P. Ojha, H. Yurtseven, A.K. Prajapati and M.I.N.M. Mohan, Liquid Crystal Research: Current Trends and Future Perspectives, Adv. In Condensed Matter Phys., 2013. (Hindawi Publishing Corporation).

14. C.C. Huang and S.C. Lien, Phys. Rev.,A31(1985)2621.

15. K. Denolf, B. Van Roie, G. Pitsi and J. Thoen, Mol. Cryst. Liq. Cryst., 449(2006)47.

16. Ch. Bahr and G. Heppke, Mol. Cryst. Liq. Cryst., 150b(1987)313.

17. B.R. Ratna, R. Shashidhar, G.G. Nair, S.K. Prasad, Ch. Bahr and G. Heppke, Phys. Rev. A, $37(1988) 1824$.

18. J. Zubia, M. Castro, J.A. Puertolas, J. Etxebarria, M.A.P. Jubindo and M.R. de La Fuente, Phys. Rev. E, 48(1993)1970. 
19. F. Mercuri, M. Marinelli, U. Zammit, C. C. Huang, and D. Finotello, Phys. Rev. E, 68(2003)051705.

20. C.C. Huang and S. Dumrangrattana, Phys. Rev. A, 34(1986)5020.

21. Ch. Bahr, G. Heppke and B. Sabaschus, Ferroelectrics, 84(1988)103.

22. B.Zeks, Mol. Cryst. Liq. Cryst., 114(1984)259.

23. R. Blinc, in "Phase Transitions in Liquid Crystals" (Plenum Press, New York 1992).

24. S. Salihoğlu, H.Yurtseven, A. Giz, D. Kayışoğlu and A. Konu, Phase Trans., 66(1998)259.

TABLE 1: Values of the Landau free-energy parameters (Eq.8) using the experimental data [3], as shown in Fig. (1) for C7.

\begin{tabular}{|c|c|l|l|l|l|c|}
\hline $\begin{array}{l}\text { Curves } \\
\text { in Fig.(1) } \\
\Theta \text { vs. E }\end{array}$ & $\begin{array}{l}a \times 10^{4} \\
\left(\mathrm{Jm}^{-3} \mathrm{~K}^{-1} \mathrm{rad}^{-2}\right)\end{array}$ & $\begin{array}{l}l \\
\left(\mathrm{Jm}^{-3} \mathrm{rad}^{-4}\right)\end{array}$ & $\begin{array}{l}\mathrm{C} \\
\left(\mathrm{Jm}^{-3} \mathrm{rad}^{-6}\right)\end{array}$ & $\mathrm{T}_{0}\left({ }^{0} \mathrm{C}\right)$ & $\chi_{0}$ & $\begin{array}{l}C \times 10^{8} \\
\left(\mathrm{JC}^{-1} \mathrm{~m}^{-1} \mathrm{rad}^{-1}\right)\end{array}$ \\
\hline $\mathrm{a}$ & 2.47 & 381 & $-5.21 \times 10^{4}$ & 54.46 & 4.2 & 1.90 \\
\hline $\mathrm{b}$ & 2.21 & -23.24 & -8.88 & 52.66 & 4.1 & 1.80 \\
\hline $\mathrm{c}$ & 2.23 & -26.26 & 114.65 & 52.46 & 4.1 & 1.78 \\
\hline $\mathrm{d}$ & 2.43 & -29.83 & 144.25 & 52.26 & 4.3 & 1.82 \\
\hline e & 2.44 & -32.67 & 166.10 & 52.06 & 4.3 & 1.82 \\
\hline f & 2.44 & -29.51 & 148.32 & 51.86 & 4.3 & 1.82 \\
\hline g & 2.44 & -36.69 & 194.91 & 51.66 & 4.3 & 1.82 \\
\hline Ref. 3 & 8.93 & $-1.34 \times 10^{6}$ & $6.57 \times 10^{6}$ & 51.36 & 4.2 & 0.858 \\
\hline
\end{tabular}

TABLE 2: Values of the coefficients for the temperature dependence of the electric displacement (D) according to Eq.(12) and the fitted parameters for the inverse tilt susceptibility $\left(\chi_{\theta}^{-1}\right)$ according to Eq.(11) for $\mathrm{T}<\mathrm{T}_{\mathrm{C}}$ and $\mathrm{T}>\mathrm{T}_{\mathrm{C}}$ using the experimental data [3] for $\mathrm{C}$.

\begin{tabular}{|c|c|c|c|c|c|c|c|c|c|}
\hline \multicolumn{10}{|c|}{$\mathrm{T}<\mathrm{T}_{\mathrm{C}}$} \\
\hline $\begin{array}{l}E \\
\left(\frac{k V}{c m}\right)\end{array}$ & $\begin{array}{l}\mathrm{T}_{\mathrm{C}} \\
\left({ }^{0} \mathrm{C}\right)\end{array}$ & $\begin{array}{l}a^{\prime} \\
\left(\mathrm{nC} / \mathrm{cm}^{2}\right)\end{array}$ & $\begin{array}{l}b^{\prime} \\
\left(\frac{n C}{\mathrm{~cm}^{2} \cdot{ }^{\circ} \mathrm{C}}\right)\end{array}$ & $\begin{array}{l}c^{\prime} \times 10^{-4} \\
\left(\frac{n C}{\mathrm{~cm}^{2} \cdot{ }^{0} \mathrm{C}^{2}}\right)\end{array}$ & $\begin{array}{l}a \times 10^{18} \\
\left(\frac{\mathrm{Jm}^{-3} K^{-1}}{\mathrm{rad}^{2}}\right)\end{array}$ & $\begin{array}{l}b \times 10^{10} \\
\left(\frac{\mathrm{Jm}^{-3}}{r a d^{4}}\right)\end{array}$ & $\begin{array}{l}c \times 10^{15} \\
\left(\frac{\mathrm{Jm}^{-3}}{r a d^{6}}\right)\end{array}$ & $\chi_{0}$ & $\begin{array}{l}C \times 10^{8} \\
\left(\frac{J C^{-1} m^{-3}}{\operatorname{rad}}\right)\end{array}$ \\
\hline 10 & 55.17 & -37.10 & 0.227 & -3.47 & -4.0 & 17.12 & $-5.7 \times 10^{7}$ & 4.57 & -8.05 \\
\hline 30 & 55.48 & -43.78 & 0.267 & -4.08 & -2.26 & 25.14 & $-2.42 \times 10^{8}$ & 4.60 & -2.21 \\
\hline 50 & 55.86 & -122.2 & 0.745 & -11.3 & -8.27 & -0.035 & 2.36 & 4.65 & 2.91 \\
\hline \multicolumn{10}{|c|}{$\mathrm{T}>\mathrm{T}_{\mathrm{C}}$} \\
\hline 10 & 55.17 & 15.24 & -0.093 & 1.41 & -2.12 & 5.82 & $-3.41 \times 10^{7}$ & 4.60 & -13.15 \\
\hline 30 & 55.48 & 66.66 & -0.405 & 6.15 & -1.81 & 2.07 & $-3.09 \times 10^{6}$ & 4.63 & -12.36 \\
\hline 50 & 55.86 & 132.18 & -0.803 & 12.2 & 1.09 & $1.13 \times 10^{8}$ & -6.91 & 4.60 & 3.22 \\
\hline
\end{tabular}

\section{FIGURE CAPTIONS}

FIGURE 1 Tilt angle $(\Theta)$ as a function of the field strength $(E)$ at various temperature differences with respect to the transition temperature $\mathrm{T}_{0}$ (Table 1) according to Eq.(8) using the experimental data [3] for C7.

FIGURE 2 Electric displacement (D) as a function of the field strength (E) at various temperature differences using the experimental data [3] as calculated according to Eq.(10) for C7. Observed values are also shown here.

FIGURE 3 Calculated values of the tilt angle $(\Theta)$ as a function of the field strength at various temperature differences (Fig. 3) using the experimental data [3] for $\mathrm{C}$.

FIGURE 4 Temperature dependence of the electric displacement (D) at various field strengths indicated using the experimental data [3] for C7.

FIGURE 5 Temperature dependence of the static dielectric constant $(\epsilon)$ at various field strengths indicated using the experimental data [3] for C7. 
Page 7 of 7

Canadian Journal of Physics
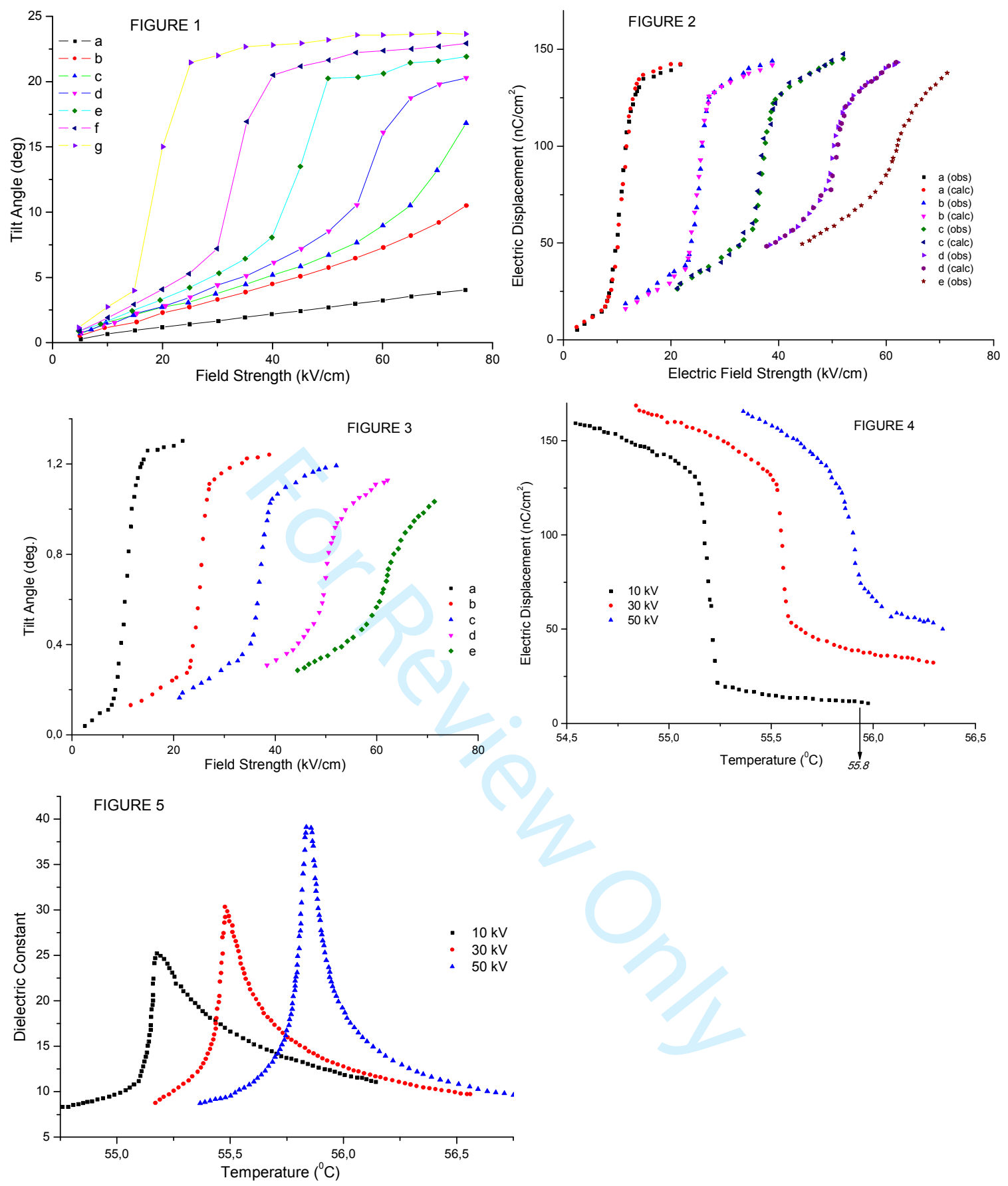

https://mc06.manuscriptcentral.com/cjp-pubs 